\title{
Study on Deep Learning as a Powerful Technology that Revolutionizing Automation in Industries
}

\author{
Manju Susan Thomas, MPhil \\ Assistant Professor, Department of Computer Science and Applications \\ SAFI Institute of Advanced Study \\ Vazhayoor, Kerala, India
}

\begin{abstract}
Smart production refers to the usage of superior records analytics to complement bodily technology for enhancing device performance and choice making. With the extensive deployment of sensors and Internet of Things, there is a growing need of managing large manufacturing facts characterized through excessive quantity, excessive velocity, and high range. Deep gaining knowledge of present's superior analytics gear for processing and analyzing huge production facts. This paper affords a comprehensive survey of typically used deep mastering algorithms and discusses their programs in the direction of making production "clever". The evolvement of deep mastering technologies and their benefits over traditional gadget gaining knowledge of are first of all mentioned. Subsequently, computational strategies based totally on deep getting to know are provided specifically purpose to improve device overall performance in manufacturing. Several consultant deep mastering models are comparably mentioned. Finally, emerging topics of research on deep learning are highlighted, and destiny trends and challenges related to deep getting to know for smart production are summarized.
\end{abstract}

Keywords: deep learning, technology, revolution, automation, industries 


\section{INTRODUCTION}

Deep learning is the field of learning deep organized and unstructured portrayal of information. Deep learning is the developing pattern in AI to digest better outcomes when information is vast and complex. Deep learning design comprises of deep layers of neural systems, for example, input layer, shrouded layers, and yield layer. Shrouded layers are utilized to comprehend the mind boggling structures of information. A neural system shouldn't be modified to play out an intricate errand. Gigabytes to terabytes

\subsection{Background of Deep Learning}

Since 2006, profound organized learning, or all the more regularly called profound learning or progressive learning, has developed as another region of AI explore. Amid the previous quite a while, the strategies created from profound learning research have just been affecting a wide scope of flag and data preparing work inside the customary and the new, extended degrees including key parts of AI and manmade brainpower; see outline articles in and furthermore the media inclusion of this advancement in .A progression of workshops, instructional exercises, and special issues or meeting special sessions as of late have been dedicated exclusively to profound learning and its applications to different flag and data preparing territories. These include:

- 2008 NIPS Deep Learning Workshop;

- 2009 NIPS Workshop on Deep Learning for Speech Recognition and Related Applications;

- 2009 ICML Workshop on Learning Feature Hierarchies;

\subsection{Automation in Industry}

Much industry is mechanized and exceptionally specialized utilizing programmed framework. Development of in enterprises is colossal and of more extensive degree. Be that as it may, mechanization level differs from industry to industry. Farming, deals and some administration industry are hard to mechanize though industry like correspondence especially phone industry its exceptionally robotized seething from managing, transmission and charging all done naturally. In rural industry is relevant in the preparing and pressing of sustenance's however there are some administration territories which can't be computerized.
Over the beyond century, the producing industry has passed through some of paradigm shifts, from the Ford meeting line (1900s) to Toyota production gadget (1960s), flexible manufacturing (1980s), reconfigurable production (1990s), agent-based production (2000s), cloud production (2010s). Various nations have evolved strategic roadmaps to transform production to take advantage of the emerging infrastructure, as presented by way of Internet of Things (IoTs) and information science. As an instance, Germany added the framework of Industry 4.Zero in 2010, which has been evolved right into a collaborative attempt amongst member international locations within the European Union. Similarly, in 2011 the Smart Manufacturing Leadership Coalition (SMLC) in the U.S. Created a systematic framework for imposing clever production. The plan "China Manufacturing 2025", delivered in China in 2015, goals to sell superior production. As manufacturing machines are more and more geared up with sensors and communication abilties, there is full-size ability to further enhance the condition-awareness of manufacturing machines and approaches, lessen operational downtime, enhance the level of automation and product great and response more well timed to dynamically converting consumer demands. Statistics suggests that $82 \%$ of the companies the usage of clever production technology have experienced increased efficiency and forty five $\%$ of the organizations of the agencies skilled elevated purchaser delight. Smart production refers to a brand new production paradigm where manufacturing machines are completely connected via wireless networks, monitored by way of sensors, and managed via advanced computational intelligence to enhance product great, device productivity, and sustainability at the same time as lowering costs. Recent advancement of Internet of Things (IoTs), Cloud Computing, Cyber Physical System (CPS) offers key assisting technology to improve contemporary production. By leveraging these new technology in manufacturing, records at distinct stages of a product's existence, ranging from uncooked materials, machines' operations, facility logistics, and even human operators, is amassed and processed . With the proliferation of producing facts, records pushed intelligence with advanced analytics transforms unparalleled volumes of data into actionable and insightful information for clever manufacturing as illustrated in Fig. 1. Data driven intelligence fashions the complex multivariate nonlinear relationships amongst facts, with no in-intensity expertise of gadget physical behaviours required. Data driven intelligence has attracted massive research effort for manufacturing records distilling and choice making. 


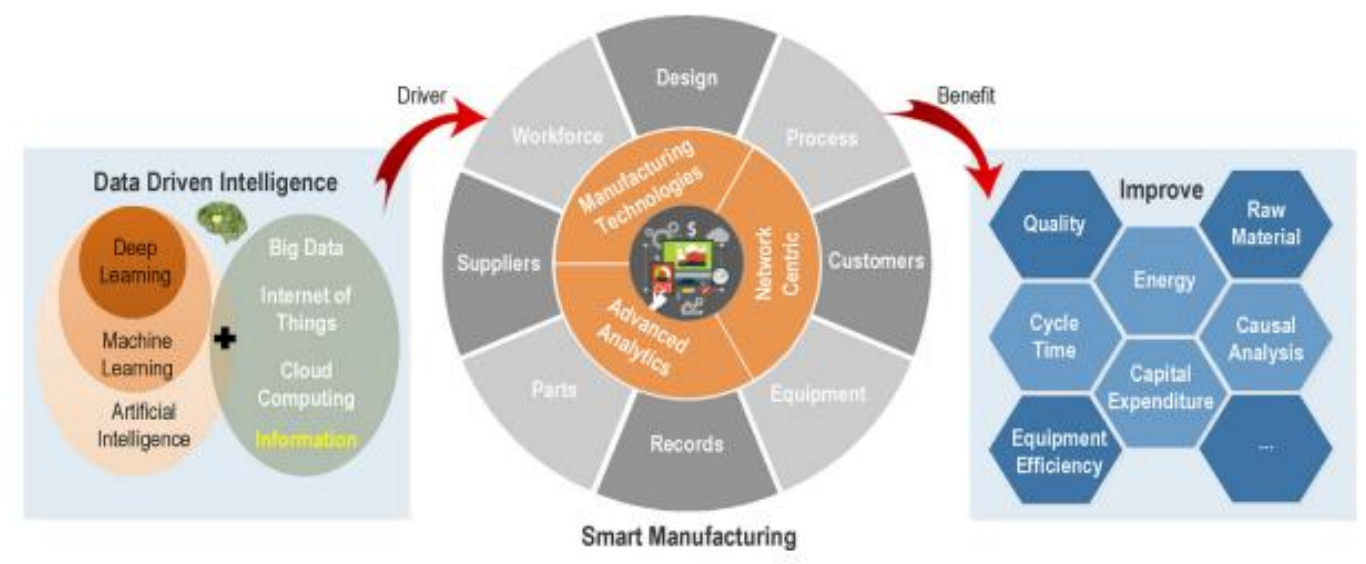

Fig. 1. The role of data driven intelligence in smart manufacturing.

In statistics mining techniques are categorized into 5 categories, together with characterization and description, affiliation, classification, prediction, clustering and evolution analysis. The obstacles to statistics-pushed choice making in manufacturing are also identified. Typical device mastering strategies are reviewed in for intelligent production, and their strengths and weaknesses also are mentioned in a wide variety of producing packages. A comparative study of device learning algorithms such as Artificial Neural Network, Support Vector Machine, and Random Forest is performed for machining device wear prediction. The schemes, strategies and paradigm of developing selection making guide structures are reviewed for the tracking of machining operations, and those strategies consist of neural networks, fuzzy common sense, genetic algorithms, and hybrid structures. The potential gain and successful software examples of usual machining studying techniques such as Bayesian Networks, instancebased gaining knowledge of, Artificial Neural Network, and ensemble techniques are mentioned in Cloud enabled analysis strategies including records driven method, physics primarily based in addition to model-based totally strategies are reviewed in, with the blessings from both advanced computing functionality and facts sharing for sensible choice making. Traditional system getting to know is generally designed with shallow structures, inclusive of Artificial Neural Network, Support Vector Machine, and logistic regression, and many others. By coping with restricted handcrafted functions, it achieves first rate overall performance in a spread of packages. However, the big information in smart production imposes a diffusion of demanding situations, inclusive of the proliferation of multimodal statistics, high dimensionality of feature space, and multicollinearity among information measurements. These challenges render traditional algorithms struggling and thus greatly hinder their overall performance. As a step forward in artificial intelligence, deep gaining knowledge of demonstrates fantastic overall performance in numerous packages of speech recognition, image recondition, herbal language processing (e.G. Translation, information, check questions \& solutions), multimodal photograph-textual content, and games (e.G. Alphago). Deep studying permits robotically processing of information toward notably nonlinear and complicated function abstraction through a cascade of multiple layers, rather than handcrafting the premiere function representation of facts with domain expertise. With automated function gaining knowledge of and excessive-extent modelling abilities, deep learning provides a sophisticated analytics tool for clever manufacturing in the huge information technology. It uses a cascade of layers of nonlinear processing to learn the representations of records corresponding to specific degrees of abstraction. The hidden styles under every other are then recognized and predicted via quit-to-quit optimization. Deep studying gives wonderful capacity to boost recordsdriven production applications, in particular inside the large records generation In mild of the above challenges, this paper objectives to provide a ultra-modern review of deep mastering strategies and their packages in clever production. Specifically, the deep gaining knowledge of enabled advanced analytics framework is proposed to fulfill the opportunistic need of clever manufacturing. The standard deep gaining knowledge of models are briefly added, and their programs to production are outlined to highlight the trendy development in applicable areas. The challenges and future traits of deep getting to know are mentioned ultimately. The rest of paper is built as follows. Firstly, data-driven artificial intelligence strategies are reviewed in Section 2, with the superiority of deep gaining knowledge of strategies outlined. Next, the challenge and opportunistic need of deep studying in smart production are offered, and standard deep mastering models are in short mentioned in Section 3. Then, the modern packages of deep learning techniques within the context of clever manufacturing are summarized in Section 4. Finally, the challenges as well as destiny developments of deep getting to know in clever manufacturing are discussed.

\section{REVIEW OF LITERATURE}

Artificial intelligence is considered as a essential manner to own intelligence, and indexed because the first vicinity in Gartner's Top 10 strategic technology tendencies in 2017 . Artificial intelligence has experienced numerous lifecycles, 
from the infancy period (Forties), via the primary upsurge duration (Sixties) and the second one upsurge period (Eighties), and the prevailing third boom duration (after 2000s). The improvement trend and standard artificial intelligence models are summarized in Table 1.The foundation of Artificial Neural Network started out lower back in 1940s, while MP version and Hebb rule were proposed to talk about how neurons worked in human brain. At the workshops in Dartmouth College, great synthetic intelligence talents like playing chess games and fixing simple logic problems have been evolved. The pioneering paintings brought artificial intelligence to the first upsurge duration (Nineteen Sixties).

In 1956, a mathematical model named Perceptron changed into proposed to simulate the apprehensive system of human getting to know with linear optimization. Next, a network version called Adaptive Linear Unit turned into advanced in 1959 and have been efficaciously utilized in practical programs which includes communication and climate forecasting. The dilemma of early artificial intelligence become additionally criticized because of the difficulty in dealing with non-linear problems, together with XOR (or XNOR) class.

With the improvement of Hopfield community circuit [28], synthetic intelligence advanced to the second one upsurge (Nineteen Eighties). Back Propagation (BP) set of rules was proposed to clear up non-linear problems in complex neural community in 1974 .A random mechanism

Table 1 List of typical artificial intelligence models

\begin{tabular}{ll}
\hline Timeline & Proposed models \\
\hline Infancy period (1940s) & MP model \\
First upsurge period & Hebb rule \\
(1960s) & Perceptron \\
Second upsurge period & Hoptive Linear Unit \\
(1980s) & Back Propagation \\
& Boltzmann Machine \\
& Support Vector Machine \\
& Restricted Boltzmann Machine \\
& Auto Encoder \\
Third boom period & Recurrent Neural Network \\
(after 2000s) & Long short-term Memory \\
& Convolutional Neural Network \\
& Deep Belief Network \\
& Deep Auto Encoder \\
& Sparse Auto Encoder \\
& Deep Boltzmann Machine \\
& Denosing Auto Encoder \\
& Deep Convolutional Neural Network \\
& Generative Adversarial Network \\
& Attention-based LSTM \\
\hline
\end{tabular}

Changed into delivered into Hopfield community and recommend the Boltzmann Machine (BM) in 1985 . With the development of statistical studying, Support Vector Machine (SVM) was advanced with kernel capabilities transformation in 1997, and showed first rate performance on category and regression. However, those traditional device studying strategies require human knowledge for feature extraction to lessen the size of enter, and as a consequence their performance exceptionally is based at the engineered features. The delivery of deep gaining knowledge of blessings now not handiest from the rich accumulation of traditional machine mastering strategies, however additionally the muse of statistical getting to know. Deep learning uses records representation studying as opposed to explicit engineered functions to perform responsibilities. It transforms data into summary representations that permit the functions to be learnt.
In 1986, Restricted Boltzmann Machine (RBM) turned into developed with the aid of obtaining the possibility distribution of Boltzmann Machine , and the hidden layers were used as characteristic vectors to represent the input statistics. Meanwhile, Auto Encoder (AE) changed into proposed using the layer-by means of-layer Greedy gaining knowledge of set of rules to reduce the loss function. In 1995, a neural network with directed topology connections among neurons, called Recurrent Neural Network (RNN), was proposed for function mastering from sequence records .In 1997, an progressed model of recurrent neural network, named Long short-term Memory (LSTM), became proposed to address the vanishing gradient hassle and cope with complex time sequence statistics. 
In 1998, Convolutional Neural Network (CNN) changed into recommend to deal with dimensional inputs (e.G. Photograph), in which capabilities gaining knowledge of have been done by way of stacking convolutional layers and pooling layers .As the hierarchical structures of deep mastering models getting deeper, version education and parameter optimization emerge as more hard and time eating, even leading to over fitting or local optimization problems. Many tries have been made to expand deep getting to know fashions, but no quality performance changed into reported earlier than 2006.

Deep Belief Network (DBN) became advanced and achieved fulfillment in 2006.It allowed bidirectional connections in top layer best instead of stacking RBMs directly to lessen computational complexity, and the parameters have been efficaciously learned via layer-smart pre-training and high-quality tuning. Meanwhile, Deep Auto Encoder turned into proposed by using including greater hidden layers to deal with high nonlinear enter . The version parameters were first off pre-trained the use of a greedy layer-with the aid of-layer unsupervised gaining knowledge of set of rules and then first-class-tuned the use of BP algorithm. One 12 months later, Sparse Auto Encoder (SAE) became placed for ward to lessen dimensionality and analyze sparse representations.

Deep gaining knowledge of gained growing popularity. In 2009, Deep Boltzmann Machine with a bidirectional structure turned into proposed to study ambiguous input facts robustly, and the model parameters had been optimized using layer-smart pre-schooling . In 2010, Denoising Auto Encoder changed into supplied to reconstruct the stochastically corrupted input information, and pressure the hidden layer to discover greater robust functions . Deep Convolutional Neural Network (DCNN) become delivered with deep structure of Convolutional Neural Network in 2012 , and it confirmed superior performance in photograph reputation. Generative Adversarial Network (GAN) turned into proposed in 2014 , and it contained two unbiased models performing as adversaries. The generative version became designed to generate random samples just like real samples while the discriminative version changed into used for training and class with each actual and generated random samples.

In 2016, an interest-based LSTM version changed into proposed by way of integrating attention mechanism with LSTM . Nowadays, more and more new models are being advanced even according to week.

\subsection{Comparison between deep learning and traditional machine learning}

Both deep gaining knowledge of and traditional device learning are records driven artificial intelligence techniques to model the complicated courting between input and output as proven in Fig. 2. In addition to the excessive hierarchical structure, deep learning also has extraordinary attributes over traditional device getting to know in phrases of feature getting to know, model production, and version training. Deep mastering integrates feature mastering and version production in a single version via selecting extraordinary kernels or tuning the parameters via stop to cease optimization. Its deep architecture of neural nets with many hidden layers is essentially multi-level non-linear operations. It transfers every layer's illustration (or functions) from original input into greater abstracted representation in the higher layers to discover the complex inherent systems. For example, the functions which includes area, corner, contour, and object components, are abstracted layer-by way of-layer from an image. These abstracted function representations are then enter to the classifier layer to perform type and regression duties. Overall, deep getting to know is an give up-to-end studying shape with the minimum human inference, and the parameters of deep getting to know version are educated together.

On the contrary, traditional system gaining knowledge of plays function extraction and version construction in a separated manner, and each module is constructed step-bystep. The hand made capabilities are first off extracted by means of reworking uncooked information right into a distinct domain (e.G., statistical, frequency, and timefrequency area) to take the representative statistics requiring expert area knowledge. Next, function choice is accomplished to enhance the relevancy and decrease the spurious redundancy amongst features before feeding into the device mastering version. Traditional gadget getting to know techniques typically has shallow structures with at most 3 layers (e.G. Input, output, and one hidden layer). Thus, the performance of the built model no longer best is predicated on the optimization of adopted algorithms (e.G. BP Neural Network, Support Vector Machine, and logistic regression), however also is heavily stricken by the handcrafted features. Generally, the function extraction and selection are time-ingesting, and highly rely upon domain information.

Therefore, deep mastering has exclusive distinction with conventional machine getting to know strategies as illustrated in Table 2. The high stage summary representation in function studying makes deep studying greater flexible and adaptable to data variety. Because the statistics are abstracted, the numerous records kinds and assets do now not have Sturdy have an impact on at the evaluation results. On the alternative hand, the deep hierarchical structure in deep gaining knowledge of is less complicated to version then on linear relationship using compositional feature evaluating with the shallow structure which is regarded as a everyday function in traditional system getting to know. The superiority of deep community have been established mathematically in. As the scale and sort of dataset grow inside the huge facts context, it will become extra difficult to create new, notably relevant features. 


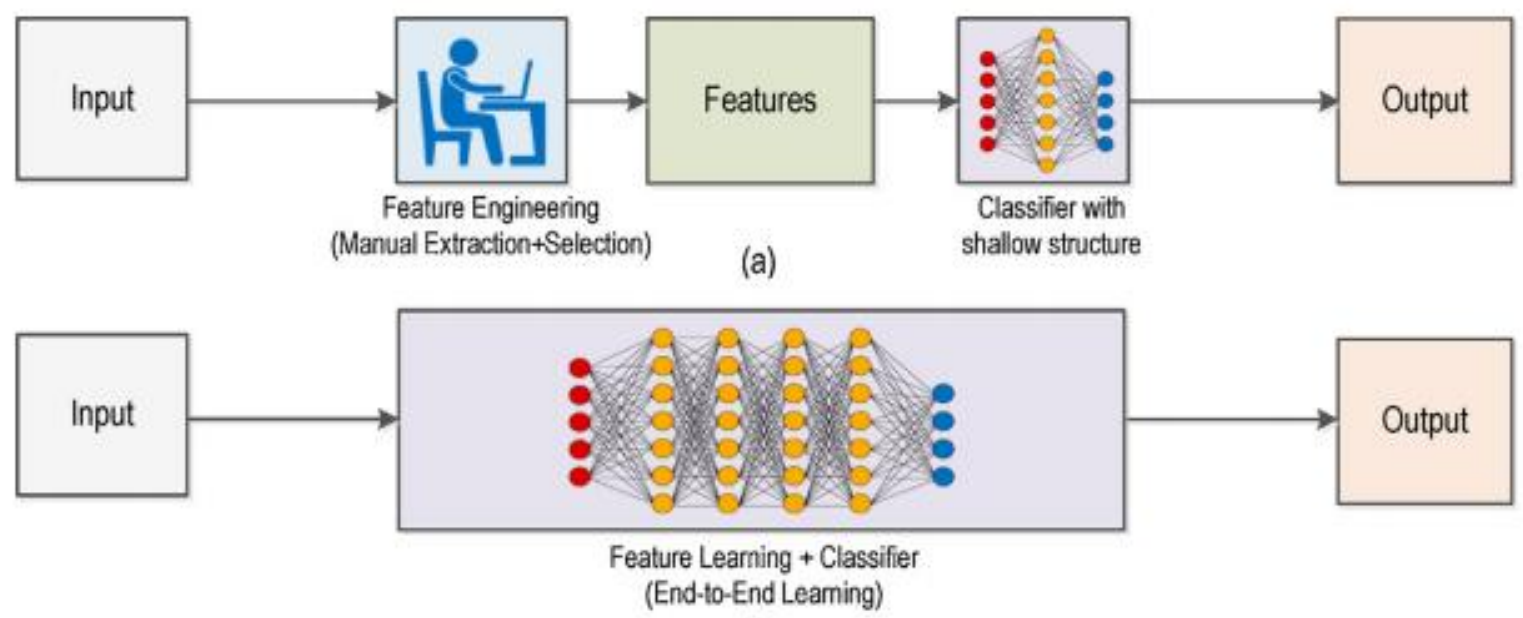

(b)

Fig. 2. Comparison between two techniques: a) traditional machine learning, b) deep learning.

Table 2 Comparison between traditional machine learning and deep learning

\begin{tabular}{llll}
\hline & Feature learning & Model construction & Model training \\
\hline $\begin{array}{l}\text { Traditional machine } \\
\text { learning }\end{array}$ & $\begin{array}{l}\text { Explicit engineered } \\
\text { features extracted with } \\
\text { expert domain knowledge. }\end{array}$ & $\begin{array}{l}\text { Use extracted features to } \\
\text { construct data-driven model, } \\
\text { usually with shallow } \\
\text { structures. }\end{array}$ & $\begin{array}{l}\text { Each module is trained } \\
\text { step-by-step. }\end{array}$ \\
Deep learning & $\begin{array}{l}\text { An end-to-end high } \\
\text { hierarchical model structure } \\
\text { with nonlinear combination of }\end{array}$ & \\
& transforming darned by & Parameters are trained jointly. \\
& abstract representations. & & \\
& & multi-layers. & \\
\hline
\end{tabular}

In the context of big information generation in smart production, the capability to keep away from function engineering is regarded as a tremendous advantage because of the demanding situations associated with this process.

\section{RESEARCH METHODOLOGY}

\subsection{Deep Studying for Smart Manufacturing}

With new technologies (e.G. IoT, massive facts) embraced in smart production, smart facilities recognition on developing manufacturing intelligence that can have a high quality effect across the complete organization. The production these days is experiencing an unprecedented boom in available sensory information comprised of various formats, semantics, and structures. Sensory information turned into accrued from distinct elements across the producing organization, inclusive of product line, production system, production manner, labor activity, and environmental situations. Data modelling and analysis are the vital a part of smart manufacturing to managing improved high volume information, in addition to assisting actual-time data processing.

From sensory statistics to manufacturing intelligence, deep mastering has attracted tons attention as a breakthrough of computational intelligence. By mining know-how from aggregated facts, deep gaining knowledge of techniques play a key role in automatically getting to know from facts, identifying patterns, and making selections as proven in Fig. Three. Different ranges of statistics analytics can be produced including descriptive analytics, diagnostic analytics, predictive analytics, and prescriptive analytics. Descriptive analytics objectives to summarize what takes place by taking pictures the product's situations, surroundings and operational parameters. When the product overall performance is reduced or the equipment failure happens, diagnostic analytics study the foundation cause and document the cause it takes place. Predictive analytics 
utilizes statistical fashions to make predictions about the possibility of future manufacturing or system degradation with available historical data. Prescriptive analytics is going beyond by using recommending one or more courses of motion. Measures can be identified to enhance production results or correct the problems, showing the likely final results of every choice.

With the superior analytics supplied through deep gaining knowledge of, production is transformed into noticeably optimized smart facilities. The advantages consist of decreasing running expenses, preserving up with converting purchaser demand, improving productivity and lowering downtime, gaining better visibility and extracting greater value from the operations for globally competitiveness.

Up to this point, diverse deep gaining knowledge of architectures had been advanced and the applicable studies subjects are rapid-growing. To facilitate the investigation of producing intelligence, numerous standard deep gaining knowledge of architectures are mentioned inclusive of Convolutional Neural Network, Restricted Boltzmann Machine, Auto Encoder, and Recurrent Neural Network and their variants. The feature learning capability and model production mechanism had been emphasized considering those models are the constructing blocks to construct complete and complex deep studying techniques.

\subsection{Convolutional neural network}

Convolutional Neural Network (CNN) is a multi-layer feed forward artificial neural community that's first of all proposed for two-dimensional picture processing .It has additionally been investigated for one-dimensional sequential information analysis such as natural language processing and speech popularity recently. In CNN, the feature studying is achieved by way of alternating and stacking convolutional layers and pooling operations. The convolutional layers convolve with uncooked enter statistics the usage of more than one neighborhood kernel filters and generate invariant nearby capabilities. The subsequent pooling layers extract the most sizeable capabilities with a set length over sliding windows of the raw enter facts by using pooling operations which includes max pooling and common pooling. Max pooling selects the most fee of 1 vicinity of the feature map as the most good sized feature. Average pooling calculates the imply cost of one vicinity and takes it as the pooling cost of this place. Max pooling is nicely appropriate to extract sparse features, whilst pooling operation on all samples may not be most desirable. After multi-layer feature gaining knowledge of, fully-linked layers convert a - dimensional function map right into a one dimensional vector and then feed it into a soft max function for version construction. By stacking convolutional layers, pooling layers, and completelyrelated layers, an ordinary $\mathrm{CNN}$ is constructed as proven in Fig. Four. Gradient based totally back propagation is normally used to educate convolutional neural network by using minimizing the minimum suggest squared mistakes or pass-entropy loss feature. CNN has the fine houses which includes sparse interactions with neighborhood connectivity, parameter sharing with reduced numbers, and equivariant illustration which is invariant to object locations. 
International Journal of Computer Applications Technology and Research

Volume 9-Issue 03, 83-97, 2020, ISSN : -2319-8656

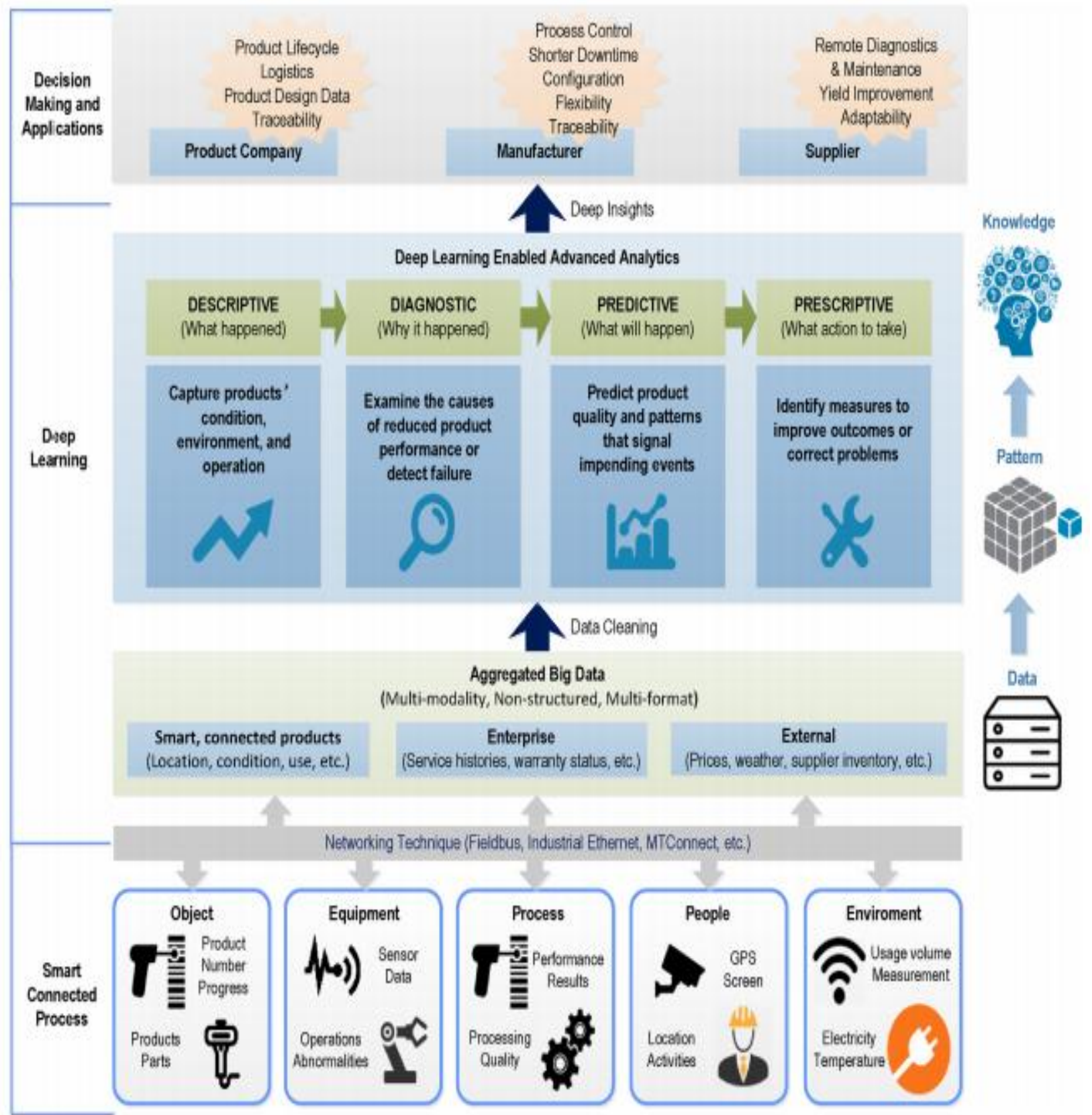

Fig. 3. Deep learning enabled advanced analytics for smart manufacturing.

\subsection{Restricted Boltzmann System and its Versions}

Restricted Boltzmann Machine (RBM) is a two-layer neural community which includes seen and hidden layer. There exists a symmetric connection between seen and hidden gadgets, however there are not any connections among every neuron inside the equal layer. It is an energy based version wherein the visible layer is used to input records at the same time as the hidden layer is used to extract functions. All hidden nodes are assumed conditionally independent. The weights and offsets of those layers are tuned over iterations so that it will make the output of the seen layer as the approximation of the unique input. Finally, the hidden layers are appeared as special representations of the seen layer. The parameters in hidden layers are handled as the capabilities to symbolize the enter records to comprehend statistics coding and size discount.Then, supervised gaining knowledge of methods which include logistic regression, Naïve Bayes, BP Neural Network, and Support Vector Machine, and many others.May be used to put into effect data class and 
regression. RBM takes the benefits of extracting required functions from schooling datasets routinely, which avoids the nearby minimal cost and for this reason has received a growing quantity of attentions. Utilizing RBM as the fundamental getting to know module, different version models had been advanced . Deep Belief Network (DBN):
DBN is built with the aid of stacking a couple of RBMs, wherein the output of the 1 th layer in hidden units is used as the enter of the $(1+1)$ th layer in visible units. For DBN schooling, a quick greedy set of rules is normally used to initialize

the network

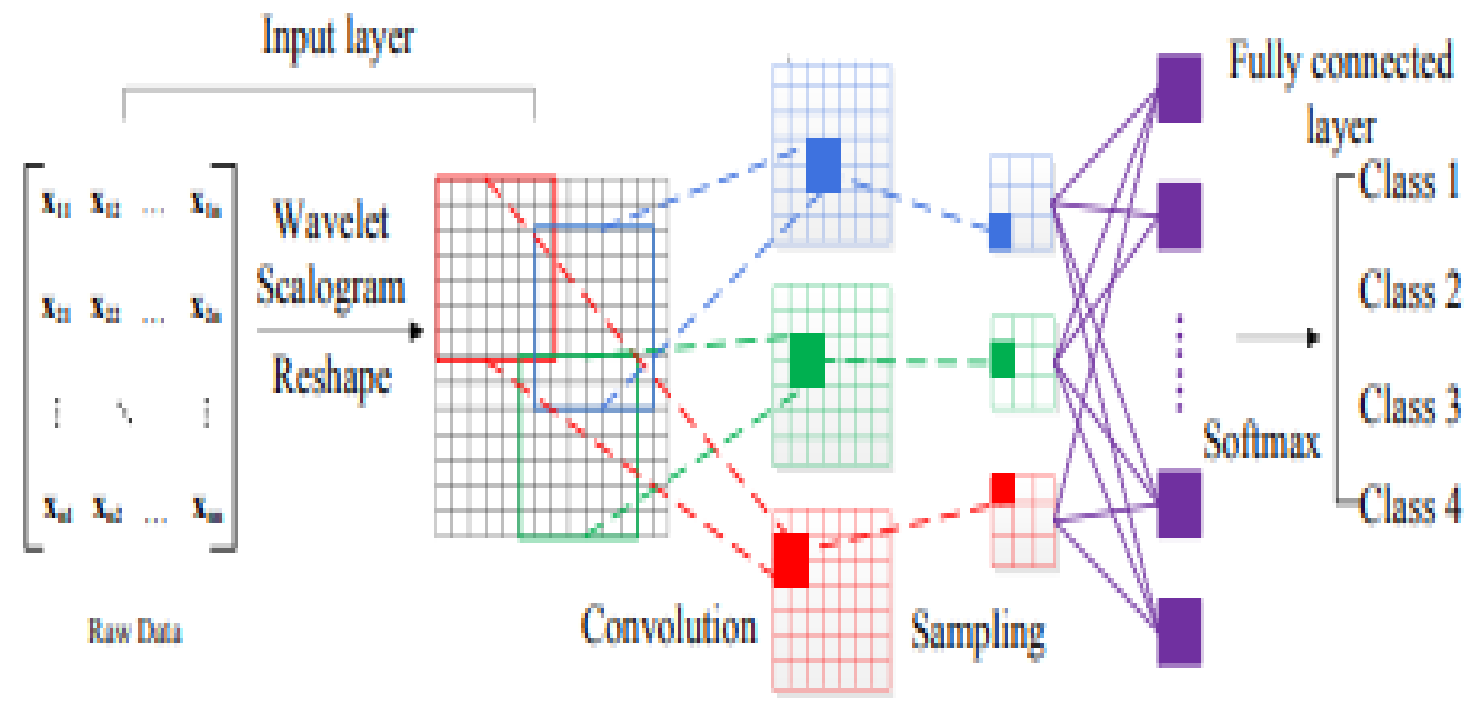

Fig. 4. Architecture of convolutional neural network model.

The parameters of this deep structure are then first-ratetuned by way of a contractive wake-sleep algorithm .Bayesian Belief Network is carried out to the location that is close to the visible layers, and RBMs are used to the area some distance from the visible layers. That is to say, the best layers are undirected and the opposite lower layers are directed, as proven in Fig. 5. Deep Boltzmann Machine (DBM): DBM can be regarded as a deep established RBMs wherein hidden devices are grouped right into a hierarchy of layers.

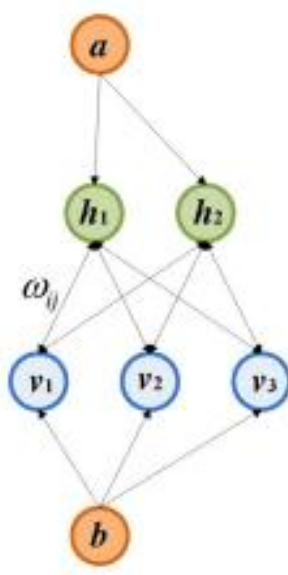

(a)

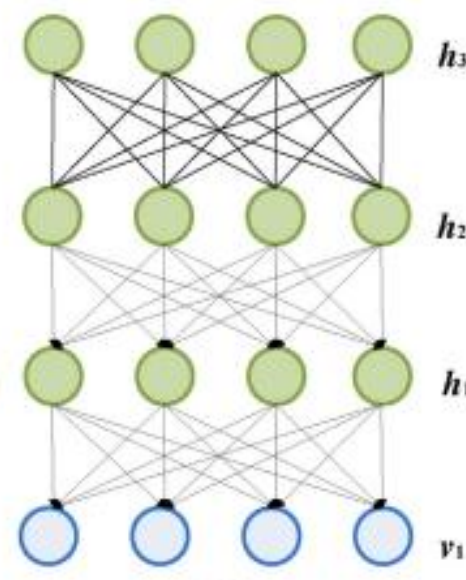

(b)

(b)

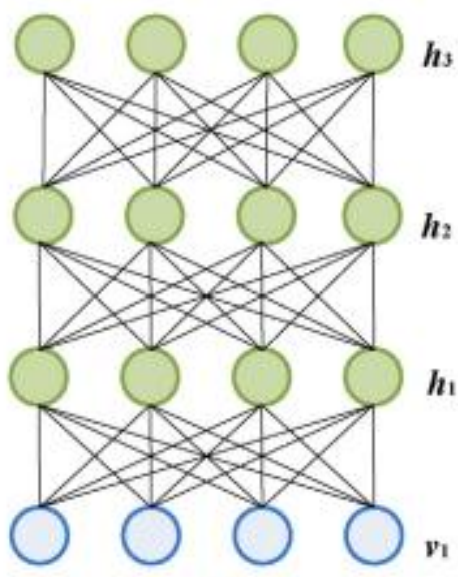

(c)

Fig. 5. Architecture of (a) RBM, (b) DBN, and (c) DBM. 
The full connections among two adjoining layers are enabled, however no connection is authorized within a layer or between non-neighbouring layers as proven in Fig. Five. By stacking multi-RBMs, DBM can research complex structures and construct excessive-stage illustration of enter information . Compared to DBN, DBM is a fully undirected graphical model even as DBN is a mixed directed/undirected one. Accordingly, the DBM model is trained together and more computationally expensive. On the opposite, DBN may be skilled layeraccurately to be extra correctly.

\subsection{Recurrent Neural Network and its Variants}

Compared with conventional neural networks, Recurrent Neural Network (RNN) has unique characteristic of topology connections among the neurons formed directed cycles for series facts as shown in Fig. 7. Thus, RNN is suitable for feature getting to know from sequence statistics. It lets in statistics persists in hidden layers and captures preceding states of some time steps ago. An updated rule is implemented in RNN to calculate the hidden states at extraordinary time steps. Take the sequential enter as a vector, the present day hidden country can be calculated through two elements thru a same activation function (e.G. Sigmoid or tan h function). The first part is calculated with the input while the second one component is acquired from the hidden country on the preceding time step. Then, the goal output may be calculated with the present day hidden country via a gentle max characteristic. After processing the whole collection, the hidden country is the learned illustration

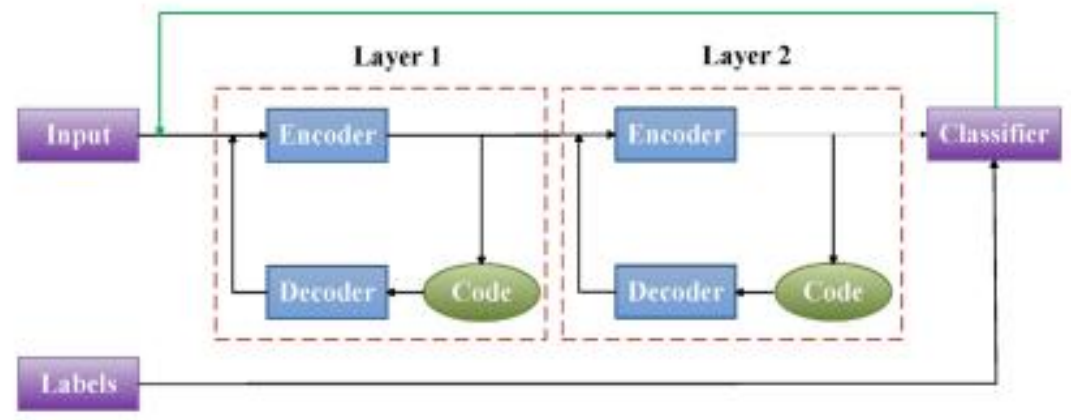

Fig. 6. The architecture of AE.

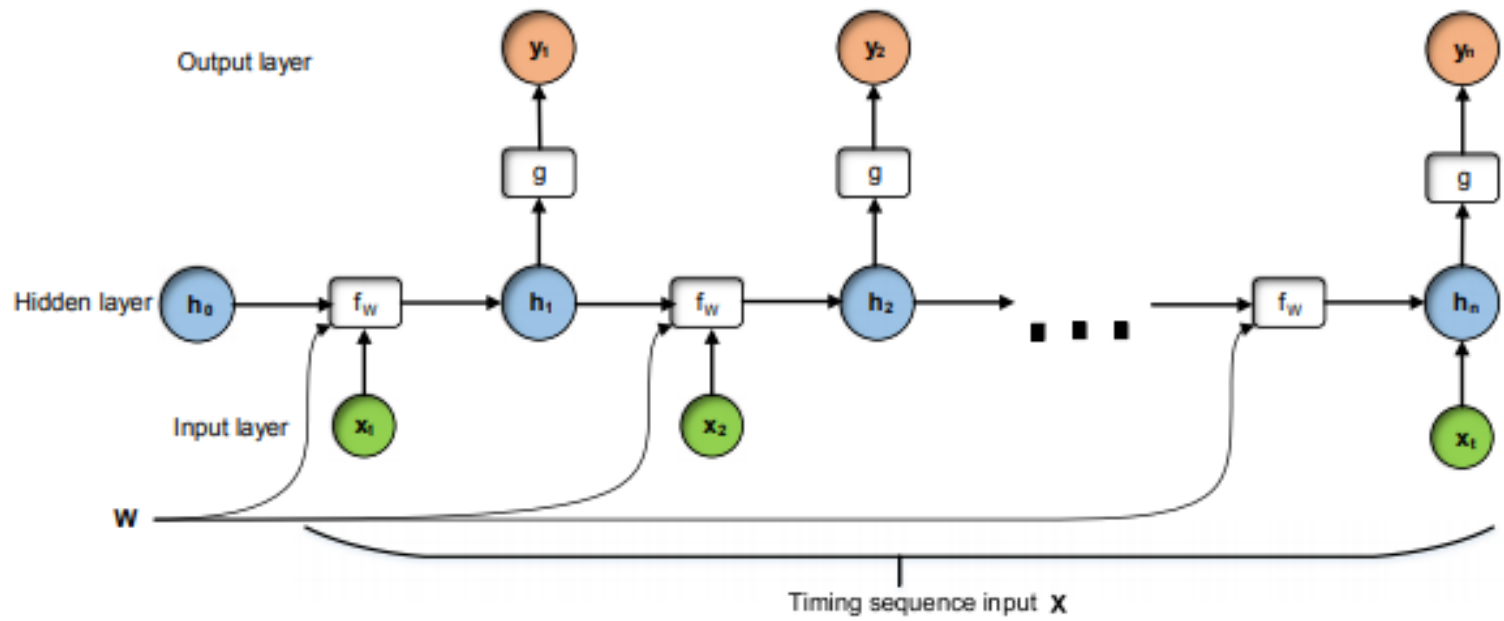

Fig. 7. Architecture of recurrent neural network model.

Of the enter sequential information and a conventional multilayer perceptron (MLP) is brought on top to map the acquired representation to objectives. Different from conventional neural networks, the version education in
RNN is done via Back propagation Through Time (BPTT). RNN is first off unrolled in step with time and every unrolled time step is taken into consideration as an additional layer. Then lower back propagation set of rules is implemented to calculate gradients. Due to the 
vanishing/exploding gradient problem using BPTT for version education, RNN can't capture long-time period dependencies. In different words, RNN has trouble in handling lengthy-time period collection facts. A sort of enhancements are proposed to remedy these troubles, amongst which lengthy quick-time period reminiscence (LSTM) is broadly investigated for its effectiveness. The most crucial concept of LSTM is cell state, which permits statistics waft down with linear interactions. Comparing with single recurrent structure in RNN, the gates including overlook gate layer, enter gate layer and output gate layer, are utilized in LSTM to govern the mobile nation. It permits every recurrent unit to adaptively seize lengthyterm dependencies of different time scales.

\section{DATA ANALYSIS}

\subsection{Applications to Smart Manufacturing}

Computational intelligence is an crucial a part of clever production to permit correct insights for higher choice making. Machine getting to know has been broadly investigated in one-of-a-kind tiers of manufacturing lifecycle overlaying concept, layout , evaluation, production, operation, and sustainment as proven in Fig. 8. The programs of data mining in manufacturing engineering are reviewed in, overlaying different categories of manufacturing processes, operations, fault detection, maintenance, selection help, and product first-rate improvement. The evolution and destiny of producing are reviewed in, emphasizing the importance of data modelling and analysis in manufacturing intelligence. The utility schemes of gadget getting to know in manufacturing are diagnosed as summarized in .Smart production also calls for prognostics and health management (PHM) skills to meet the current and destiny needs for efficient and reconfigurable production. Deep learning, as an rising technique, has been investigated for a extensive variety of producing systems recently. To supply a top level view, the applications of latest deep mastering techniques in manufacturing are discussed on this have a look at, especially inside the areas of product nice inspection, fault analysis, and disorder analysis, as highlighted in Table.

Table 3 Comparison between different deep learning models.

\begin{tabular}{|c|c|c|c|}
\hline Model & Priniple & Pros. & Cons. \\
\hline CNN & $\begin{array}{l}\text { Abstracted features are leanned } \\
\text { by stacked convolutional and } \\
\text { sampling layers. }\end{array}$ & $\begin{array}{l}\text { Reduced parameter number, } \\
\text { invariance of shift, scale and } \\
\text { distortion }\end{array}$ & $\begin{array}{l}\text { High computational } \\
\text { complexity for high } \\
\text { hierarchical model training }\end{array}$ \\
\hline RBM & $\begin{array}{l}\text { Hidden layer describes variable } \\
\text { dependencies and connections } \\
\text { between input or output layers } \\
\text { as representative features. }\end{array}$ & $\begin{array}{l}\text { Robust to anbiguous input and } \\
\text { training label is not required in } \\
\text { pre-training stage }\end{array}$ & $\begin{array}{l}\text { Time-consuning for joint } \\
\text { parameter optinization }\end{array}$ \\
\hline $\mathbb{A}$ & $\begin{array}{l}\text { Unsupervised feature larning } \\
\text { and data dimensionality } \\
\text { reduction are achieved through } \\
\text { encoding }\end{array}$ & $\begin{array}{l}\text { Irrelevane in the input is } \\
\text { eliminated, and meaningul } \\
\text { information is proserved }\end{array}$ & $\begin{array}{l}\text { Error propagation } \\
\text { layer-by-layer and sparse } \\
\text { representations are not } \\
\text { guaranteed }\end{array}$ \\
\hline RNN & $\begin{array}{l}\text { Temporal pattern stored in the } \\
\text { fecurrent neuros connection } \\
\text { and distributed hidden states } \\
\text { for time-series data. }\end{array}$ & $\begin{array}{l}\text { Short-term information is } \\
\text { retained and temporal } \\
\text { correlations are captured in } \\
\text { sequence data. }\end{array}$ & $\begin{array}{l}\text { Dificult to train the model and } \\
\text { suve the long-term dependence }\end{array}$ \\
\hline
\end{tabular}


International Journal of Computer Applications Technology and Research Volume 9-Issue 03, 83-97, 2020, ISSN : -2319-8656

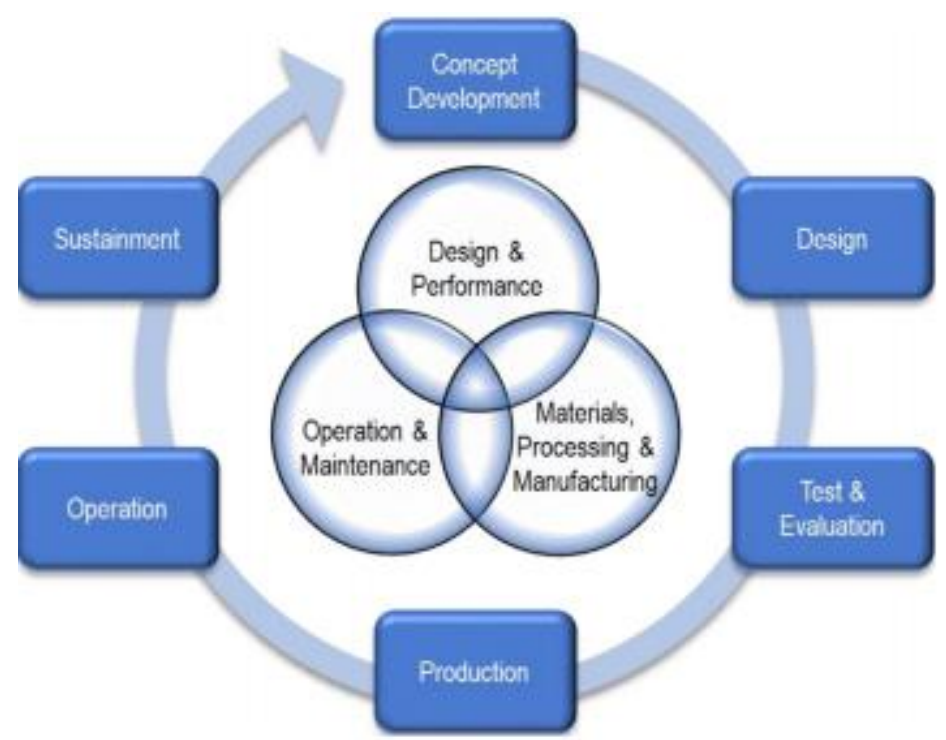

Fig. 8. Typical application scenarios of machine learning in smart manufacturing. 


\subsubsection{Descriptive analytics for product high-quality inspection}

Surface integration inspection is commonly inspected using device imaginative and prescient and picture processing techniques to come across Table 5 A list of deep mastering fashions with packages. Deep studying version Application Scenarios Reference CNN Surface integration inspection [Machinery fault analysis DBN Machinery fault prognosis Predictive analytics \& disorder prognosis AE Machinery fault diagnosis RNNs Predictive analytics \& disorder diagnosis surface illness for superior product first-rate in production . Traditional system gaining knowledge of has made exquisite development and yields dependable consequences in lots of instances ,but exceptional pre-processing procedures inclusive of structural-based, statistical based, filter out-based, and version based totally techniques are needed to extract consultant capabilities with expert know-how . However, flexible configuration in current manufacturing gadget may want to shift manufacturing from one product to every other quick. The function illustration may need redesign from scratch for classic gadget learning. Additionally, a brand new product can also gift complex texture patterns or intensity variations, and the floor defects could be in an arbitrary length, orientation and form. Therefore, manually designed capabilities in conventional device mastering technique might also lead to inadequate or unsatisfactory inspection overall performance in complicated surface scenarios or dynamic changing technique. To deal with these challenges, deep getting to know has been investigated to examine excessivestage regular capabilities and implemented to a huge variety of textures or tough-to-locate defects instances. Convolutional Neural Network, firstly designed for photo analysis, is nicely in shape for automatic illness identity in surface integration inspection. In , a Deep Convolutional Neural Network architecture is designed and the hyper-parameters are optimized based totally on back propagation and stochastic gradient descent algorithms. A max-pooling Convolutional Neural Network is offered in to perform characteristic extraction directly from the pixel illustration of metallic disorder pix and shows decrease error costs comparing with multi-layer perceptron and guide vector gadget. The image evaluation is studied with convolutional neural community in to robotically look at dirties, scratches, burrs, and wears on floor parts. The experimental consequences display that $\mathrm{CNN}$ works well with one-of-a-kind styles of defects on textured or non-textured surfaces. A popular technique based on CNN is proposed in to extract patch characteristic and are expecting disorder place thru thresholding and segmenting. The results display the pertained CNN version works well on small dataset with advanced accuracy for computerized floor inspection device.

\subsubsection{Predictive analytics for defect prognosis}

In order to boom production productiveness at the same time as lowering upkeep cost, it's far crucial to increase and implement an wise maintenance strategy that permits manufacturers to decide the condition of in-service systems on the way to predict when upkeep need to be done. The temporal behaviour in the historical facts is critical for prediction, and deep recurrent neural community has proven its capability to model temporal pattern. Recently, a popular recurrent neural network, named lengthy short time period memory, has been investigated to are expecting disorder propagation and estimate final beneficial lifestyles (RUL) of mechanical systems or additives. In,a competitive gaining knowledge of-based RNN has been proposed for lengthy-time period analysis of rolling bearing fitness repute. In,a new nearby characteristic-primarily based gated recurrent unit network has been proposed to learn the representation of the sequence of nearby features and the proposed approach is validated on 3 real gadget fitness tracking tasks. In , an incorporated approach of CNN and bi-directional LSTM is presented for machining device wear prediction, in which $\mathrm{CNN}$ is used to extract nearby capabilities from sequential signals and bi-directional LSTM to capture long-term dependence for prediction. Vanilla LSTM is investigated in to estimate the closing beneficial existence of an plane turbofan engine below complex operating situations and sturdy history noise, and the experimental effects verify that Vanilla LSTM affords good prediction accuracy. A stacked LSTM community allows the getting to know of higher level temporal functions, and has been presented for anomaly prediction of space trip and engine.

Deep Belief Network, as the feature getting to know approach in regression models, has also been investigated for predictive analytics. Deep Belief Network is investigated to version the complex relationship between cloth elimination fee and chemical mechanical polishing method parameters in semiconductor manufacturing. An integrative technique of Deep Belief Network and particle clear out is offered in for the RUL prediction of a ceramic bearing. By aggregating the output of ensemble DBNs, Support Vector Regression model is investigated to are expecting electricity load call for . To expect the useful resource request in cloud computing, DBN is proposed in to optimize task agenda and stability the computational load.

\subsubsection{Data matter}

As the evolution of clever manufacturing, increasingly more machineries are geared up with smart sensors and meshed with Internet of Things. Currently, most companies do not understand what to do with the information they've, and that they lack software and modelling to interpret and analyse them. On the other hand, producers want practical steerage to improve their tactics and products, while the academics increase updated artificial intelligence fashions with out considering how they'll be carried out in exercise. As manufacturing technique turns into extra complicated, extra problem comes along to clean the records and formulate the right troubles to version. Five gaps are diagnosed in clever production innovation together with adopted techniques, progressed information collection, use and sharing, predictive model design, generalized predictive fashions, and related factories and manage tactics. To meet the excessive call for of superior analytics in clever production, deep gaining knowledge of with feature learning and deep network gives terrific capacity and indicates positive residences. To handle overwhelming statistics characterised with the aid of highquantity, highvelocity and excessive-range,there are nonetheless some demanding situations associated with production enterprise to undertake, put in force, and deploy deep learning for actual-international programs. To deal with the challenges, the destiny improvement trends of deep gaining knowledge of for clever production are discussed in phrases of records remember, version selection, version visualization, prevalent version, and incremental learning. 
A common presumption in device mastering is that algorithms can research better with more statistics, and thus the overall performance of deep mastering version heavily relies upon on the dimensions and excellent of datasets. So some distance deep mastering indicates the effectiveness when it's far carried out to limited varieties of statistics (e.G. Pics, speech, and vibration, and many others.) and properly-described responsibilities. Multi-sensory has been instrumented to capture statistics at all ranges of a product's lifestyles. Deep mastering algorithm can be infeasible to immediately deal with such excessive dimensional, multimodality, and nondependent statistics, or even at risk of the curse of dimensionality. Extracting the relevant facts to lessen the dimensions and making use of appropriating mission-unique regularization time period may additionally enhance the performance of deep learning. On the other hand, the magnificence imbalance trouble is some other undertaking. The magnificence follows a noticeably-skewed distribution in real existence, representing maximum facts samples belong to few categories. For example, the dataset of floor defects is typically too small and steeply-priced to collect. The ratio of correct to horrific elements is fairly imbalanced ranging from nine: 1 to even much less than $1,000,000$. Thus, it's far tough to use widespread type techniques to differentiating precise elements from scraps. Appropriate measures along with elegance re-sampling, cost-touchy training, and integration of boot strapping may be essential for deep studying model to address magnificence imbalance troubles

\subsubsection{Incremental learning}

The deep getting to know algorithms aren't basically constructed to analyze incrementally and are consequently vulnerable to the facts speed troubles. For a new trouble setup, deep gaining knowledge of might also want to rebuild the version from scratch and the existing knowledge may be difficult to make use of. Additionally, the facts within the new event ualities is likewise an problem. It is essential to permit deep studying with incremental gaining knowledge of talents. Transfer studying targets to extract the knowledge from one supply task after which applies the found out know-how to a extraordinary but related undertaking .It could rent the pretrained deep learning model from a relevant task for version initialization and first-class-tuning to enable understanding reuse and updating as transferred deep learning. Some preceding works focusing on transferred function extraction/dimensionality reduction were done. A most imply discrepancy (MMD) degree evaluating the discrepancy among supply and goal domains is introduced into the target characteristic of deep neural networks .Thus, transferred deep learning is meaningful and promising for clever manufacturing to permit expertise updating and intelligence upgrading.

\section{CONCLUSION}

Deep getting to know provides superior analytics and gives remarkable potentials to smart manufacturing inside the age of massive information. By unlocking the unparalleled amount of facts into actionable an in sightful facts, deep gaining knowledge of gives choice-makers new visibility into their operations, as well as actual-time overall performance measures and costs. To facilitate advanced analytics, a comprehensive assessment of deep gaining knowledge of strategies is offered with the programs to smart production.
Four usual deep mastering models along with Convolutional Neural Network, Restricted Boltzmann Machine, Auto Encoder, and Recurrent Neural Network are mentioned in element. The emerging studies effort of deep gaining knowledge of in applications of manufacturing is likewise summarized. Despite of the promising results pronounced up to now, there are nevertheless a few barriers and substantial demanding situations for in addition exploration. As the evolution of computing sources (e.G., cloud computing, fog computing, etc.), computational intelligence including deep gaining knowledge of can be push into cloud, allowing extra convenient and on-call for computing offerings for smart manufacturing.

\section{ACKNOWLEDGEMENTS}

Manju Susan Thomas deeply acknowledge the encouragement received from the management of SIAS, Vazhayoor.

\section{REFERENCES}

1. Putnik G, Sluga A, ElMaraghy H, Teti R, Koren Y, Tolio T, et al. Scalability in manufacturing systems design and operation: state-of-the-art and future developments roadmap. CIRP Ann Manuf Technol 2013;62(2):751-74.

2. Lee YT, Kumaraguru S, Jain S, Hatim Q, Robinson S, Helu M, et al. A classification scheme for smart manufacturing systems' performance metrics. Smart Sustain Manuf Syst 2017;1(1):52-74.

3. $\mathrm{Hu} \mathrm{T}$, Li P, Zhang C, Liu R. Design and application of a real-time industrial Ethernet protocol under Linux using RTAI. Int $\mathbf{J}$ Comput Integr Manuf 2013;26(5):429-39.

4. Ye Y, Hu T, Zhang C, Luo W. Design and development of a CNC machining process knowledge base using cloud technology. Int $\mathrm{J}$ Adv Manuf Technol 2016:1-13.

5. Tao F, Qi Q. New IT driven service-oriented smart manufacturing: framework and characteristics. IEEE Trans Syst Man Cybern Syst 2017;99:1-11.

6. Ang J, Goh C, Saldivar A, Li Y. Energyefficient through-life smart design, manufacturing and operation of ships in an industry $\quad 4.0$ environment. Energies 2017;10(5):610.

7. Huang Z, Hu T, Peng C, Hou M, Zhang C. Research and development of industrial realtime Ethernet performance testing system used for CNC system. Int J Adv Manuf Technol 2016;83(5-8):1199-207.

8. Lalanda P, Morand D, Chollet S. Autonomic mediation middleware for smart 
manufacturing. IEEE Internet Comput 2017;21(1):32-9.

9. Smart Manufacturing Coalition. Manufacturing growth continues despite uncertain economy, according to ASQ outlook survey; 2013. https:// smartmanufacturingcoalition.org/sites/default/f iles/12.16.13 manufacturing outlook survey.pdf. [Accessed 10 Sepember 2017].

10. Wang L, Törngren M, Onori M. Current status and advancement of cyber-physical systems in manufacturing. J Manuf Syst 2015;37:517-27.

11. Wang P, Gao RX, Fan Z. Cloud computing for cloud manufacturing: benefits and limitations. J Manuf Sci Eng 2015;137:1-10.
12. Lu Y, Xu X, Xu J. Development of a hybrid manufacturing cloud. J Manuf Syst 2014;33(4):551-66.

13. Wu D, Rosen DW, Schaefer D. Cloud-based design and manufacturing: status and promise. Comput Aided Des 2015;59:1-14.

14. Choudhary AK, Harding JA, Tiwari MK. Data mining in manufacturing: a review based on the kind of knowledge. J Intell Manuf 2009;20(5):501-21.

15. Lade $P$, Ghosh R, Srinivasan S. Manufacturing analytics and industrial internet of things. IEEE Intell Syst 2017;32(3):74-9. 